\title{
Affect School for chronic benign pain patients showed improved alexithymia assessments with TAS-20
}

\author{
Eva O Melin ${ }^{+1,2,3}$, Hans O Thulesius ${ }^{*}+1,2,4$ and Bengt A Persson ${ }^{\dagger 5,6}$
}

\begin{abstract}
Background: Alexithymia is a disturbance associated with psychosomatic disorders, pain syndromes, and a variety of psychiatric disorders. The Affect School (AS) based on Tomkins Affect Theory is a therapy focusing on innate affects and their physiological expressions, feelings, emotions and scripts. In this pilot study we tried the AS-intervention method in patients with chronic benign pain.

Methods: The AS-intervention, with 8 weekly group sessions and 10 individual sessions, was offered to 59 patients with chronic non-malignant pain at a pain rehabilitation clinic in Sweden 2004-2005. Pre and post intervention assessments were done with the Hospital Anxiety and Depression scale (HAD), the Toronto Alexithymia Scale-20 (TAS20), the Visual Analogue Scale for pain assessment (VAS-pain), the European Quality of Life health barometer (EQoL) and the Stress and Crisis Inventory-93 (SCl-93). After the group sessions we used Bergdahl's Questionnaire for assessing changes in interpersonal relations, general well-being and evaluation of AS.

Results: The AS intervention was completed by 54 out of 59 (92\%) patients. Significant reductions in total TAS-20 posttest scores $(p=0.0006)$ as well as TAS-20 DIF and DDF factors (Difficulties Identifying Feelings, and Difficulties Describing Feelings) were seen ( $p=0.0001$, and $p=0.0008$ ) while the EOT factor (Externally Oriented Thinking) did not change. Improvements of HAD-depression scores $(p=0.04)$, EQoL $(p=0.02)$ and self-assessed changes in relations to others ( $p<0.001$ ) were also seen. After Bonferroni Correction for Multiple Analyses the TAS-20 test score reduction was still significant as well as Bergdahl's test after group sessions. The HAD, EQoL, SCI-93, and VAS-pain scores were not significantly changed. The AS-intervention was ranked high by the participants.
\end{abstract}

Conclusions: This pilot study involving 59 patients with chronic benign pain indicates that the alexithymia DIF and DDF, as well as depression, social relations and quality of life may be improved by the Affect School therapeutic intervention.

\section{Background}

\section{Alexithymia}

The alexithymia construct includes difficulties to identify and describe feelings, difficulties in distinguishing between feelings and the bodily sensations of emotional arousal, constricted imaginative processes and an externally oriented cognitive style [1]. Sifneos first described the dysfunction in 1967, and in 1972 the term alexithymia was introduced [2]. According to Nemiah and Sifneos a

\footnotetext{
* Correspondence: hans.thulesius@ltkronoberg.se

1 Family Care Centre Strandbjörket, Kronoberg County Council, SE-351 85

Växjö, Sweden

+ Contributed equally

Full list of author information is available at the end of the article
}

deficit in the capacity for symbolization of emotions results in a variety of manifestations including abnormal physiological reactions, a propensity for impulsive behaviour, discomfort with and avoidance of social relationships, and an impaired capacity for self-care and selfregulation [3]. The limited ability to process emotions cognitively by experiencing them as conscious feelings leads both to amplification of the somatic sensations accompanying emotional arousal, and/or to physical reactions as immediate responses to unpleasant arousal [1]. The autonomous reactions may also be enhanced by physiological dysregulation [4-6].

( 2010 Melin et al; licensee BioMed Central Ltd. This is an Open Access article distributed under the terms of the Creative Commons BHoWed Central Attribution License (http://creativecommons.org/licenses/by/2.0), which permits unrestricted use, distribution, and reproduction in any medium, provided the original work is properly cited. 
Alexithymia is commonly found in people suffering from psychosomatic and psychiatric disorders including chronic pain patients (prevalence 35-47\%) [7-9], hypochondria, substance abuse, eating disorders [1], panic disorder [10] and depression [1,11-13]. In recent years it is debated whether alexithymia is a stable personality trait $[11,14,15]$, or state dependent $[12,13]$. Particularly the relation between alexithymia and depression is controversial. If alexithymia is a personality trait this would imply that alexithymia increases the risk of becoming depressed. If alexithymia is state dependant then depression would lead to alexithymic features. In depressive states there are cognitive failures like impairment of memory functions [16]. Thus, the cognitive failure of handling emotions while depressed could be part of a general cognitive impairment.

Interpersonal relationships are difficult to deal with for individuals with alexithymia because of lacking emotional comprehension and expression. Therefore, alexithymia might be associated with reduced social support [17-19], that has been considered a protective factor in determining both the development and prognosis of disease and health problems [20,21]. Many psychotherapists claim that alexithymia is particularly difficult to treat with psychotherapy [22-24]. However, in cognitive behaviour therapy (CBT) for treating depression, the therapy outcome was not hindered by alexithymia [25].

\section{Tomkins Affect Theory}

There are four basic definitions in Tomkins Affect Theory (TAT): Affect, feeling, emotion and script. Affects are the innate, unconscious and genetically pre-programmed biological portions of emotions. Each innate affect has a specific program lasting a few seconds involving face mimicry, body gestures, voice, and autonomous nervous and hormone system physiology [26-30]. The innate affects are enjoyment-joy, interest-excitement, surprisestartle, fear-terror, anger-rage, distress-anguish, shamehumiliation, distaste, dissmell and pain. Awareness of an affect is defined as a feeling [26-30]. Emotions are affects intertwined with memory. A triggered affect evokes memories of earlier situations, relationships and scenes where this affect has been triggered before, and in addition, other affects triggered in the earlier situations will be triggered again in the present situation. An emotion lasts as long as memory continues to trigger the affect. Emotions can be a combination of both unconscious affects and conscious feelings. Scripts are learned patterns to handle emotions. Mood is defined as a persistent state of emotion [26-30]. Affects are important messengers to the self. According to TAT we all have basic drives essential for survival. The primary functions of the innate affects are to regulate these drives. The secondary functions are to regulate other affects [26-30].

\section{Pain}

Pain is defined as an unpleasant sensory and emotional experience associated with actual or potential tissue damage, or described in terms of such damage [31]. According to TAT, pain has qualities typical for both drives and affects. Pain is equal to hunger and other basic drives, while necessary for our survival. Pain seen as a drive can be amplified by affects of distress and fear. Pain seen as an affect can regulate all drives; amplify other affects like distress, anger and fear, and reduce affects of enjoyment and interest. Pain induces impaired functions at many levels that activate shame [26-30].

A definition of Chronic Pain Syndrome (CPS) according to Sanders et al [32] involves

a) Persistent pain of at least three months duration consistent with or significantly out of proportion to physical findings. At least two of the criteria b) to e) should also be present: b) progressive deterioration in ability to function at home, socially, and at work, c) progressive increase in health care utilization (such as repeated physical evaluations, diagnostic tests, requests for pain medications, and invasive medical procedures), d) demonstrated mood disturbance, and e) clinically significant anger and hostility.

Physical Symptom Disorder (PSD) is proposed to replace pain disorder together with other somatoform disorders as a diagnostic entity, whenever one or more physical symptoms are currently present, and not fully explainable by other medical or psychiatric disorders [33]. Medically unexplained symptoms were shown to be closely correlated to the alexithymic features difficulties identifying feelings and difficulties describing feelings, but not to externally oriented thinking [34]. The resolution of unexplained physical symptoms is associated with a short duration of symptoms and few physical symptoms at baseline [35]. Central sensitization is associated with chronic benign pain and leads to a reduction in pain threshold, an amplification of pain responses and a spread of pain sensitivity to non-injured areas [36].

\section{Purpose of study}

The main purpose of this study was to evaluate the psycho-educational method Affect School (AS) for participants with chronic benign pain. Would they accept the AS method and benefit from the intervention? Could it cause any harm? We also wanted to investigate a possible connection between alexithymia and the severity of selfrated anxiety, depression, and stress symptoms. A secondary purpose was to evaluate self rated acquired changes in social relations and general well-being, and 
participants' subjective feelings about having gone through an overall change.

\section{Methods}

\section{Affect School}

AS is based on TAT [26-30] and was constructed by Armelius and Bergdahl [37-39]. Goals of AS are to identify, differentiate and verbally express emotions, and to identify bodily expressions of affects in order to gain better health [37-40]. The AS method was associated with a significant reduction of self-rated anxiety, somatizing, depression and obsessive-compulsive symptoms in primary care patients [40], and reduction of stress and psychological symptoms in employees with chronic stress [37]. The AS comprises 8 weekly meetings of a 5-7 participants' group with two instructors. Each session has a special theme when one or two innate affects are discussed. The instructors systematically teach participants about the innate affects and their physiological expressions emphasizing that emotions are useful signs of one's inner states. At every session participants are encouraged to recall a specific occasion when they experienced the affect being discussed, and to express in detail how that affect was sensed in body and mind, which means that they are encouraged to tell single-event autobiographical memory (ABM) narratives, which is important in psychotherapy [41-44]. Participants then learn how to link affects with memories and specific situations. They also get general knowledge of affects, feelings, emotions and scripts. After eight group meetings each participant is offered individual Script Analysis (SA) treatment on ten occasions [37-39].

AS instructors involved in this study were one psychologist, one physician, one physiotherapist, one social counsellor, one nurse with psychotherapy training, and one occupational therapist. All instructors had a 40-hour AS training, led by Bergdahl and Persson. The first group had alternating instructors but the rest of the groups had the same two instructors during the whole program. The six instructors and three psychologists - not responsible for the group intervention - were engaged in the SA second part of the AS intervention.

\section{Subjects}

Participants were recruited within patients with chronic benign pain admitted to a Centre of Pain Rehabilitation (CoPR), during the years 2003-2005. All participants fulfilled criteria "a","b" and "c" of the CPS definition [32]. All of them also fulfilled the criteria of PSD [33]. On average, at admittance patients suffered from chronic benign pain for seven years. Fifty five participants (93\%) were on full time sick leave or full time temporary disability pension, and $4(7 \%)$ were on part time sick leave or temporary disability pension. All of them had a progressive increase in health care utilization. Thirteen participants (22\%) took antidepressant medication at admission. Participants were selected whenever the treatment team considered that they would both benefit from AS, and not influence the group process in any harmful way. All but a few participants received the AS intervention after the usual CoPR rehabilitation program.

\section{Procedures}

Psychiatric symptoms were assessed by means of selfreport tests, while somatic diagnoses were taken from medical records. Participants were given five self-report instruments before and after the whole intervention. One self-report instrument was used after the AS but before the SA together with the participants' assessments of the instructors and the group intervention. The group dynamics were observed by the instructors.

\section{Self report instruments applied before and after the whole} intervention

1) 20-item version of the Toronto Alexithymia Scale,

TAS-20 The TAS-20 is a self-report scale developed by Bagby et al [45-48] and is based on three factors: F1: DIF, F2: DDF and F3: EOT. The instrument consists of 20 statements graded from one to five. A sum of 61 points or more indicates alexithymia, a sum of 52-60 indicates an intermediate zone, while 51 points or below indicates non-alexithymia. The validity of the three factor structure has been demonstrated in several translated versions [48-51], including the Swedish version [52]. TAS-20 has been compared to the Modified Beth Israel Hospital Psychosomatic Questionnaire (Modified BIQ) which is an observer scale, and concurrent validity of the two tests was found in different cultures $[45,46,51,53]$. Also the Bermond-Vorst Alexithymia Questionnaire (BVAQ) has shown internal consistency and concurrent validity with TAS-20 [45].

Reference values for Swedish students of psychology (n $=161)$ are: mean $(\mathrm{SD})=41.6(9.2)$ for the TAS-20 total scores; mean $(\mathrm{SD})=15.1$ (4.6) for F1 (DIF); mean (SD) = 11.1 (3.7) for F2 (DDF); mean (SD) = 15.4 (3.8) for F3 (EOT) [52]. Cronbach's alpha internal reliability coefficient for the Swedish version is 0.83 for TAS-20 Global score, 0.79 for DIF, 0.77 for DDF, 0.67 for EOT [52]. The significance of the factor scales has shown divergent results [6,54-56].

\section{2) Hospital Anxiety and Depression Scale, HAD The}

HAD-test was constructed as a screening instrument to obtain information about anxiety and depression in patients with somatic complaints in medical wards. Questions about symptoms that could be signs of somatic disease were thus avoided. The test consists of 7 statements reflecting anxiety (HAD-A) and 7 statements reflecting depression (HAD-D). Each statement has four response alternatives with scores from 0 to 3 . Maximum 
score for each seven-item subscale is 21 . A sum of 0-7 points for either HAD-A or HAD-D indicates no anxiety or depression, 8-10 points indicates mild anxiety or depression, 11-14 points indicates moderate anxiety or depression, and 15-21 points indicates severe anxiety or depression $[57,58]$. In the present study scores $\geq 8$ points were considered as cut-off for depression and anxiety. In a Swedish general population sample $(\mathrm{n}=624)$ HAD-A mean (SD) was 4.6 (3.7), and HAD-D mean (SD) was 4.0 (3.5) [59]. For all patients admitted to the CoPR within the same 2003-2005 period ( $\mathrm{N}=414)$, HAD-D mean (SD) was 9.0 (4.1), and median (iqr) was 9.0 (6.0;12.0). HAD-A mean (SD), in turn was 9.5 (4.8), and median (iqr) was 9.8 $(6.0 ; 13.0)$.

3) Visual Analogue Scale for pain, VAS-pain The VASpain, as used here, is a $100-\mathrm{mm}$ visual analogue scale (VAS) on a horizontal line with the descriptor "no pain" at the left end, and the "worst possible pain" at the right end. Participants are asked to mark on the line the point that they feel represent their current state. Zero means there is no pain and 100 the "worst possible pain" [60]. At the beginning of this investigation the participants estimated their VAS-pain together with a "pain matcher" - an instrument giving the patients a small electrical current through the finger. However many participants refused this "pain matcher"; what made us stop using it, thus increasing the VAS-pain response rate.

4) Modified version of European Quality of Life health barometer, EQoL The EQoL is a 100-mm VAS assessing health-related quality of life. We used a version placed horizontally, and labelled at the left side "My general health is as bad as possible", and at the right side "My general health is as good as possible" [61].

5) Stress and Crisis Inventory-93, SCI-93 With scores ranging 0-4, the SCI-93 inventory consists of 35 questions about stress symptoms divided into four groups: a) muscular symptoms secondary to the action of the sympathetic portion of the autonomous nervous system; b) other autonomous symptoms; c) hormonal symptoms; and d) two questions about memory and ability to concentrate.

Maximum score is 140 . Normal score is $0-25$ points, 26-50 points indicate "mild" stress, $51-75$ points indicate "moderate" stress, 76 points or more points indicate "severe" stress. Cut off considered in this study was $\geq 42$ points. Reference values for the Swedish normal population are mean $(\mathrm{SD})=27.7$ (11.0) for men; and mean (SD) $=30.2$ (12.0) for women [62].

\section{Assessment after group sessions}

Bergdahl's Questionnaire The questionnaire contains 3 statements concerning improved or impaired social relations;

a) close relations, b) working colleagues, c) other people; and two other questions concerning general well- being and overall change. The scale is graded from minus two to plus two, where minus two represents maximal unwanted change, zero represents no change, and plus two the maximal wanted change. The number of participants who estimated positive change was compared to the number who estimated negative changes.

The participants also assessed the instructors and the AS group sessions by responding to 14 statements on a Likert scale. Here the scale was graded from one to five, where five represented the optimal degree for 13 statements.

\section{Observations made by instructors during group}

sessions Observations were made by the instructors about spontaneous complaints, the group process and participant's ability to tell single-event ABM narratives [41].

Statistics Though the means differed little from the medians, the use of median, instead of mean values, was chosen to calculate results of the intervention because of the small sample size. Median follow-up scores were compared to baseline scores by means of the Wilcoxon Signed Ranks Test, which was computed by using SPSS for Windows version 14.0. One Way Anova, Post Hoc Tests, and multiple comparisons Bonferroni (MCB) were made when the correlations between alexithymia and anxiety, depression and stress related symptoms were calculated before intervention. Linear Regression models were adopted to evaluate if improvements on TAS-20scores and its DIF and DDF factors depended on the improvement in HAD-Depression scores. The self estimated changes in Bergdahl's questionnaire were analyzed with the Binomial Test.

Ethical approval The Regional Ethics Committee at Linköping University approved of conducting this research, Dnr: 203/04, January 25, 2005.

\section{Results}

Fifty-four out of 59 participants (92\%) completed the intervention, see Table 1 for age and gender distribution. At baseline, 36\% of participants scored 61 or above on the TAS-20; indicating a high prevalence of alexithymia; high scores on mood disturbance such as symptoms of depression, anxiety or a combination of both were noted in 52 (88\%) participants; self assessed stress symptoms above a 42 cut-off point were seen in $92 \%$ (54) of the participants (Table 1). Participants ranked high the group intervention as well as the instructors (Table 2). Participants who scored higher on the TAS-20 also had significantly higher scores on depression, anxiety and stress symptom, as compared with those with normal scores at baseline (Table 3).

The follow-up post-test questionnaires were returned by 46 out of 54 participants (85\%) completing the intervention (Additional file 1: Table S1). Significant improve- 
Table 1: Baseline characteristics of the 59 participants.

\begin{tabular}{lc}
\hline Mean age, years (range) & $46(27-64)$ \\
& N of participants \\
Women & $52(88 \%)$ \\
Men & $7(12 \%)$ \\
& \\
& \\
Anxiety & Mood and alexithymia \\
Depression & $47(80 \%)$ \\
Combined anxiety and depression & $37(63 \%)$ \\
No anxiety/depression & $32(54 \%)$ \\
Alexithymia & $7(12 \%)$ \\
Alexithymia, intermediate score & $21(36 \%)$ \\
No alexithymia & $15(25 \%)$ \\
& $23(39 \%)$ \\
Fibromyalgia & \\
Myofascial syndrome & \\
Whiplash associated disorder & \\
Lumbago ischias & $25(42 \%)$ \\
Other pain & $15(25 \%)$ \\
\hline
\end{tabular}

ments were seen post intervention in alexithymia, depression, and quality of life scores. After Bonferroni Correction for multiple analyses the change in TAS-20 scores remained significant $(\mathrm{p}=0.004)$. While the DIF and DDF factors of the TAS-20 improved significantly, the EOT did not. After the intervention 10 out of 15 participants (67\%) no longer scored above the suggested cutoff point for alexithymia (Figure 1). According to regression analyses, the observed decrease in TAS-20 global score as well as in the DDF score was independent of the diminished depression; whereas $11 \%$ of the variance in the DIF score may be explained by changes in depression (Table 4).
Table 2: Participants' assessments of the AS and group instructors. Max score 5 (*optimal score 3).

\begin{tabular}{ll}
\hline & Md(iqr) \\
Affect School as a whole & $4(1)$ \\
Instructors were warm towards the patients & $5(0)$ \\
Instructors were interested in the patients & $5(0)$ \\
Instructors were active & $5(1)$ \\
Instructors were directing (a lot: 5, a little: 1) & $3(1)^{*}$ \\
Instructors were competent & $5(0)$ \\
Instructors were supporting & $5(1)$ \\
Instructors could help & $4(1)$ \\
Instructors understood and accepted patients. & $5(1)$ \\
Instructors told about own experiences & $5(1)$ \\
Participants appreciated instructors. & $5(0)$ \\
Participants dared to show feelings & $4(1)$ \\
Participants and instructors had goals in common & $5(2)$ \\
Cooperation between Patients and instructors & $5(0)$ \\
\hline
\end{tabular}

Six out of the 8 non-respondents at follow up had scores above the cut-off point for alexithymia, before intervention. Among the 8 follow-up non-respondents, 3 of them mentioned lack of energy to participate because of divorce, disease or death in the family; 3 other said they disagreed with or simply disliked the instructors; and the other 2 failed to give any reasons for not responding.

After the intervention 5 out of 27 participants $(\mathrm{p}=0.04$ for changes in median values) no longer scored above the cut-off for depression; and 13 out of 36 ( $p=0.11$ for changes in median values) no longer scored above the cut-off point for anxiety (Figure 2).

Even after Bonferroni Correction for Multiple Analyses, Bergdahl's test of self-estimated changes in attitudes towards close relations and relationships with other people (working colleagues excluded) improved significantly; as did general well-being and the overall wanted change (Table 5).

Table 3: Correlations between anxiety, depression, stress symptoms and alexithymia. *One Way Anova, Post Hoc Tests, (MCB).

\begin{tabular}{|c|c|c|c|c|c|}
\hline & Non alexithymia Mn(SD) & Non alexithymia Md(iqr) & Alexithymia Mn(SD) & Alexithymia Md(SD) & p-value* \\
\hline Anxiety score (HAD-A) & $7.9(3.7)$ & $9.0(6.5 ; 11)$ & $11.3(3.2)$ & $11(8.5 ; 14)$ & 0.006 \\
\hline Depression score (HAD-D) & $7.1(3.6)$ & $6(5.5 ; 10.5)$ & $11.2(4.4)$ & $12(8 ; 14)$ & 0.003 \\
\hline Stress score & $55.2(19.1)$ & $53(45.5 ; 75.5)$ & $74.4(20.3)$ & $74(62 ; 83)$ & 0.006 \\
\hline (SCl-93) & & & & & \\
\hline
\end{tabular}


Table 4: Linear regression analyses of TAS-20 scores and DIF and DDF factors with change in depression scores as independent variable. ${ }^{*}=p<0.05$

\begin{tabular}{|c|c|c|c|}
\hline & TAS-20- difference & DIF-difference & DDF-difference \\
\hline $\mathrm{R}$ & 0.17 & $0.33^{*}$ & 0.13 \\
\hline R Square & 0.03 & $0.11 *$ & 0.02 \\
\hline
\end{tabular}

Observations of the group process by instructors

In the first group we had alternating instructors but since group members began to complain, the same instructors were kept throughout the whole AS group intervention for the remaining nine groups. Participants were in general restrained and sceptical during the first session; but a little more talkative during the second session and a strong engagement among participants was noted in the third session. After this third session many participants commented that sessions were too short and eventually many other mentioned they would rather have the group intervention to continue after the eight sessions. Interactions occurred frequently among participants. A bias to tell general ABM narratives was noticed, particularly in the beginning [41] and repeated reminders from the instructors were necessary to help participants to tell single-event ABM narratives. Many participants had remarkable difficulties to remember any occasion when they had felt the affect joy, and many gave narratives of major traumas when fear was the affect being discussed. Since some of the participants were dominating, while others were shyer and quiet, an important and sometimes

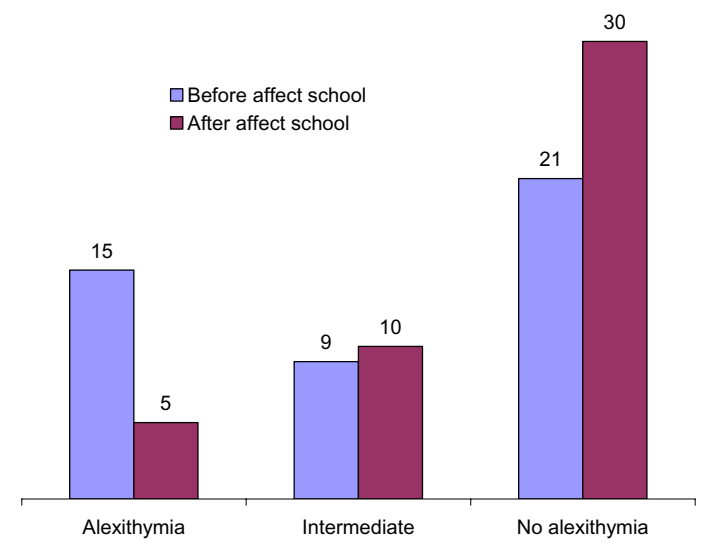

Figure 1 Number of participants with alexithymia, intermediate values and no alexithymia before and after Affect School intervention $(\mathrm{N}=45)$.

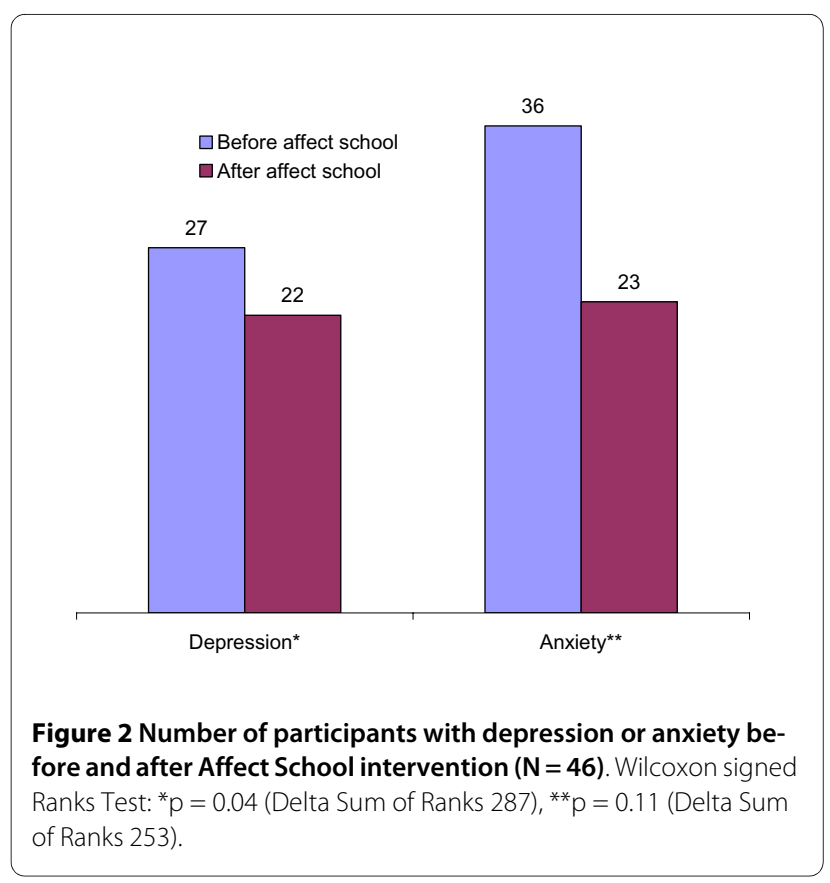

difficult task for the instructors was to balance the groups.

\section{Discussion}

Acceptance of the intervention method

This study includes a group of 59 patients with chronic pain who went through an Affect School (AS) therapeutic intervention. Since $91 \%$ of the participants completed the AS and the instructors and the AS received high evaluation scores, we may consider this an adequately accepted method of intervention. We also observed a decrease in scepticism and increased engagement during the course of the intervention, which further suggests good acceptance.

\section{Alexithymia}

The TAS-20 questionnaire has been widely validated and used all over the world, both in clinical situations and in research $[45,46,48-52,56]$. The $36 \%$ prevalence of possible alexithymia pre-intervention in our study was in a range as high as it has been described in other studies of patients suffering from chronic benign pain [7-9]. The lowering of the TAS-20 global score, as well as of the DDF score, was independent of the decrease in depression scores. Theoretically, the connection between alexithymia and depression can be understood both as a trait and as a state-dependent phenomenon; our results don't support the view of alexithymia only as state-dependent. During AS sessions, participants worked through identifying and describing affects and emotions. This corresponds to the DIF and DDF factors of the TAS-20 that showed improved post-test scores, while the factor EOT 
Table 5: Self assessed changes after termination of group sessions.

\begin{tabular}{lccccc}
\hline & N & Missing & Pos change & Neg change & p-value ${ }^{*}$ \\
\hline Close relations & 48 & 5 & 32 & 1 & $*$ \\
Relation to work colleagues & 41 & 12 & 1 & 23 & NS \\
Relations to other people & 47 & 6 & 22 & 6 & $*$ \\
General well-being & 48 & 5 & 36 & 3 & $* * *$ \\
Overall change & 47 & 6 & & $*$ \\
\hline
\end{tabular}

"After correction for multiple analyses, ${ }^{*}=p<0.05 ; * * *=p<0.001$. NS= Non significant.

was unchanged. Based on the results of no changes of EOT even after AS, there is a possibility that EOT is a more trait-prone factor than DIF and DDF. Recent Japanese data show positive correlation between EOT and Constrictive Imaginal Capacities (CIC) [56]. It would be interesting to add therapies like expressive art to the AS in order to evaluate if it also is possible to reduce EOT while achieving improved imaginal capacities.

\section{Depression and anxiety}

As the HAD test is designed to assess mood disorders among patients who seek help for somatic complaints it probably is a good test for a population of chronic pain patients. Other tests, using emotional words and requiring participants to self-rate emotions and moods can give misleading results; particularly among patients with difficulties in identifying emotions. In this study the HAD scores of anxiety and depression pre-intervention were twice as high as for a Swedish general population sample [59]. These baseline scores did not differ between the intervention group and the whole patient population admitted to the CoPR during the same period. Mood disturbances are important factors in CPS, which is also the case for the majority of the patients in our study. After the intervention our patients had a significant decrease in HAD depression scores, which did not remain after Bonferroni correction for multiple analyses. After the intervention more patients also scored below the cut-off for HAD- anxiety, but those with higher scores post intervention had a more pronounced change in their scores, than did those scoring lower. One of the aims of this study was to find out if the AS method could somehow cause any harm. To be aware of one's inner feelings can raise the anxiety levels; which may explain why some of the patients did get higher anxiety scores after the intervention. Maybe a prolonged period of SA and psychological support should be offered to patients with signs of increased mood disturbances. In a previous AS intervention a significant improvement of both anxiety and depression scores was found [40].

\section{Pain}

We have no definite answer to why we did not observe improvements in the pain parameter. The explanation can reside in a variety of problems concerning $\mathrm{A}$ ) assessment B) emotional factors or C) neurological factors. A) There are several problems with pain assessment. There are only pre and post-intervention scores concerning 38 of the 54 participants who completed the intervention. We do not know if the results would be better or worse if more had participated in the VAS-assessments. As pain is defined as an unpleasant sensory and emotional experience usually associated with actual or potential tissue damage, or described in terms of such damage [31], we would probably do better having two types of instruments; one instrument assessing the emotional facet of pain and another one just for the sensory part. We tried but failed to assess the sensory part with the so-called pain matcher. The VAS-scale was insufficient for our purpose and we do not know to what extent we were assessing the emotional or sensory part of pain. B) Emotional factors may have influenced the results. For nearly all participants, the rehabilitation period was supposed to be over after the intervention. Maybe participants hoped for more help, thus declaring that they still had significant pain, the actual pain level here somehow representing the suffering with separation from the supportive staff at the rehabilitation centre. Participants may have thought that "all pain is more or less unbearable", and as long as they felt any pain at all, they estimated it as high. The fear of the "pain matcher" might also have influenced the level of pain at follow-up as these assessments were done together initially. C) Neurological factors can also contribute to explain the lack of improvements in pain assessment. Long standing pain can be more or less reversible, but it can also require longer intervention periods due to neurological changes as in central sensitization. In fact it has already been shown that the resolution of physical symptoms depends on the duration and number of symptoms [35]. All our participants had typically suffered for a long time.

\section{Stress}

At baseline $91 \%$ of participants had high scores on stress symptoms. The mean scores of SCI-93 were beyond twice as high as the mean score in a general Swedish population sample [62]. Participants within the alexithymia group had significantly higher SCI-93 test scores than did 
those in the non-alexithymia group. However, the test does not tell us if the symptoms of stress were objectively more severe, or if the tolerance to stress related symptoms was lower in the alexithymia group. Other reports suggest that the autonomous reactions may be exaggerated and prolonged due to physiologic dysregulation [46]. The higher stress scores in the alexithymia group may thus simply reflect that their symptoms were more severe. There was no significant change in stress symptoms after the intervention, which is a different result from what Bergdahl et al [37] reported, getting less stress symptoms in employees after the AS intervention.

\section{Social relations and quality of life}

There was a statistically significant improvement of selfrated experiences of close social relationships and relations with others. As previously mentioned, interpersonal relationships are difficult to handle by persons with alexithymia. This can prove to be of major importance while impacting quality of life and health. In the group part of the intervention the participants were trained to be aware of both their own and of other people's emotions. Even though the pain scores did not improve, the EQoL scores, in turn, did. Decreased depression, improved social relations and improved self-understanding may all have a share contributing to the improved quality of life.

\section{Affect School Method - most important features}

The AS method is a structured intervention based on a well defined basic theory, TAT, and combines didactic treatment and emotional-cognitive therapy. The AS can be applied by instructors without prior psychotherapy training. Yet, a 40 hour special training was added to the basic professional competence of the instructors in our study. In every group session each participant was encouraged to tell specific, single event ABM narratives which is regarded as important in other therapies like CBT [41], Emotion-Focused Therapy (EFT) [43], and Client-Centered Therapy (CCT) [44]. The group situation is important as it helps the participants to be aware of own as well as others' emotions; what may, in turn, lead to improved social relationships. The instructors emphasize that affects are important messengers to oneself and all affects are allowed and accepted - an important feature of the AS method.

\section{Limitations}

To assess the effect of an intervention without a comparison group has indeed limitations. Yet, our patients' self defined problem was pain, and not any emotional issue. Thus we did not know, to start with, if they would even accept to participate in 18 sessions talking about affects, feelings and emotions. For that reason we had no comparison group. Patients knew they were participating in a research for a new approach to treatment, and a subjectexpectancy effect towards the intervention could thus be expected. It also remains the possibility of an observerexpectancy effect. Yet, scores for alexithymia were reduced more than scores for health related quality of life and depression, while no improvement was observed for pain or anxiety. This outcome specificity suggests a possible effect of the AS intervention over alexithymia, particularly on what concerns its DIF and DDF facets. Although TAS-20 is an instrument well validated in many cultures for both clinical and research situations, our research did nevertheless benefit from a multi-method assessment combining different observer rated as well as self-rated approaches.

\section{Conclusions}

In this pilot study we report of an educational intervention called Affect School directed towards patients with chronic non-malignant pain. The intervention was accepted by most participants. Improvements were seen regarding, depression and general health. Alexithymia features - DIF and DDF - and social relations were significantly improved even after correction for multiple analyses. Pain levels and stress symptoms were not affected.

\section{Additional material}

Additional file 1 Table S1. Scores of self-rated instrument for respondents at both baseline and follow up.

\section{Competing interests}

EOM was one of the responsible physicians at the CoPR and one of the instruc tors of the Affect School. The other authors declare that they have no competing interests.

\section{Authors' contributions}

EOM conceived and designed the study, collected and analyzed the data, and drafted the manuscript. BAP and HOT contributed to study design, and in drafting and revising the manuscript. All three authors gave final approval of publication.

\section{Acknowledgements}

We are indebted to one of the Affect School instructors, psychologist and psychotherapist Ralph Svensson. He understood the importance of emotions in the rehabilitation process for patients with chronic benign pain at an early stage. Ralph also proposed the questionnaires used in the study. We are also indebted to Anna Lindgren, PhD and Per Nyberg, PhD, for their statistical skills. This study was partially funded by the Research and Developing Fund of the County Council of Kronoberg.

\section{Author Details}

'Family Care Centre Strandbjörket, Kronoberg County Council, SE-351 85 Växjö, Sweden, 2Unit of Research and Development, Box 1223, SE-351 12 Växjö, Sweden, 3Department of Clinical Sciences Lund, Lund University, Sweden, ${ }^{4}$ Department of Clinical Sciences Malmö, Lund University, Sweden,

5Department of Psychiatry, Kronoberg County Council, PO Box 1223, SE-35112 Växjö, Sweden and ${ }^{6}$ Department of Psychology, Växjö University, SE-351 95 Växjö, Sweden

Received: 12 August 2008 Accepted: 4 June 2010 Published: 4 June 2010 


\section{References}

1. Taylor GJ, Bagby RM, Parker JD: The alexithymia construct. A potential paradigm for psychosomatic medicine. Psychosomatics 1991, 32:153-64.

2. Sifneos PE: The prevalence of "alexithymic" characteristics in psychosomatic patients. Psychother Psychosom 1973, 22:255-262.

3. Nemiah JCSP: Affect and fantasy in patients with psychosomatic disorde London UK: Butterworths; 1970.

4. Martin JB, Pihl RO: Influence of alexithymic characteristics on physiological and subjective stress responses in normal individuals. Psychother Psychosom 1986, 45:66-77.

5. Lane RD, Ahern GL, Schwartz GE, Kaszniak AW: Is alexithymia the emotional equivalent of blindsight? Biol Psychiatry 1997, 42:834-44.

6. Wehmer F, Brejnak C, Lumley M, Stettner L: Alexithymia and physiological reactivity to emotion-provoking visual scenes. J Nerv Ment Dis 1995, 183:351-7.

7. Mendelson G: Alexithymia and chronic pain: prevalence, correlates and treatment results. Psychother Psychosom 1982, 37:154-64.

8. Postone N: Alexithymia in chronic pain patients. Gen Hosp Psychiatry 1986, 8:163-7.

9. Celikel FC, Saatcioglu O: Alexithymia and anxiety in female chronic pain patients. Ann Gen Psychiatry 2006, 5:13.

10. Galderisi S, Mancuso F, Mucci A, Garramone S, Zamboli R, Maj M: Alexithymia and cognitive dysfunctions in patients with panic disorder. Psychother Psychosom 2008, 77:182-188.

11. Luminet O, Bagby RM, Taylor GJ: An evaluation of the absolute and relative stability of alexithymia in patients with major depression. Psychother Psychosom 2001, 70:254-60.

12. Saarijarvi S, Salminen JK, Toikka TB: Alexithymia and depression: a 1-year follow-up study in outpatients with major depression. J Psychosom Res 2001, 51:729-33.

13. Honkalampi K, Hintikka J, Laukkanen E, Lehtonen J, Viinamaki H: Alexithymia and depression: a prospective study of patients with major depressive disorder. Psychosomatics 2001, 42:229-34.

14. Salminen JK, Saarijarvi S, Aairela E, Tamminen T: Alexithymia--state or trait? One-year follow-up study of general hospital psychiatric consultation out-patients. J Psychosom Res 1994, 38:681-5.

15. Salminen JK, Saarijarvi S, Toikka T, Kauhanen J, Aarela E: Alexithymia behaves as a personality trait over a 5-year period in Finnish general population. J Psychosom Res 2006, 61:275-8.

16. Hinkelmann K, Moritz S, Botzenhardt J, Riedesel K, Wiedemann K, Kellner $M$, et al:: Cognitive Impairment in Major Depression: Association with Salivary Cortisol. Biol Psychiatry 2009, 25:25.

17. Kirmayer L: Languages of suffering and healing: Alexithymia as a social and cultural process. Transcul psychiatr Res Rev 1987, 24:119-136.

18. Kojima M, Senda Y, Nagaya T, Tokudome S, Furukawa TA: Alexithymia, depression and social support among Japanese workers. Psychother Psychosom 2003, 72:307-14.

19. Fukunishi I, Rahe RH: Alexithymia and coping with stress in healthy persons: Alexithymia as a personality trait is associated with low social support and poor responses to stress. Psycho Rep 1995, 76:1299-1304.

20. Lea Georg: Social support and the outcome of major depression. Br J Psychiatry 1989, 154:147-156.

21. Cohen SWTA: Stress, social support and the buffering hypothesis. Psychol Bull 1985, 98:310-357.

22. Taylor GJ, Bagby R, Parker J: Disorders of affect regulation: Alexithymia in medical and psychiatric illness Cambridge: Cambridge University Press; 1997.

23. Krystal $\mathrm{H}$ : Alexithymia and psychotherapy. Am Journal of Psychother 1979, 33:17-31.

24. Krystal $\mathrm{H}$ : Alexithymia and the effectiveness of psychoanalytic treatment. Int J Psychoanal Psychother 1982, 9:353-78.

25. Spek V, Nyklicek I, Cuijpers P, Pop V: Alexithymia and cognitive behaviour therapy outcome for subthreshold depression. Acta Psychiatr Scand 2008, 118:164-7.

26. Tomkins SS, Karon BP: Affect, imagery, consciousness: The positive affects New York: Springer; 1962.

27. Tomkins SS, Karon BP: Affect, imagery, consciousness. Vol. 2, The negative affects New York: Springer; 1963

28. Tomkins SS, Karon BP: Affect, imagery, consciousness: The negative affects, anger and fear New York: Springer Publ; 1991.
29. Tomkins SS, Karon BP: Affect, imagery, consciousness. Vol. 4, Cognition: duplication and transformation of information New York: Springer; 1992.

30. Nathanson DL: Shame and pride: affect, sex, and the birth of the self New York; London: Norton; 1992.

31. Merskey H: The definition of pain. Eur Psychiatr 1991, 6:153-159.

32. Sanders S: Clinical practice guidelines for chronic non-malignant pain syndrome patients II: An evidence-based approach. J Back Musculoskelet Rehabil 1999, 13:47-58

33. Kroenke K: Physical symptom disorder: a simpler diagnostic category for somatization-spectrum conditions. J Psychosom Res 2006, 60:335-9.

34. Deary I, Scott S, Wilson J: Neuroticism, alexithymia and medically unexplained symptoms. Person.individ. Diff 1997, 22:551-564

35. Cea Kooiman: Alexithymia does not predict the persistence of medically unexplained physical symptoms. Psychosom Med 2004, 66:224-232.

36. Ji RR, Kohno T, Moore KA, Woolf CJ: Central sensitization and LTP: do pain and memory share similar mechanisms? Trends Neurosci 2003, 26:696-705.

37. Bergdahl J, Larsson A, Nilsson LG, Ahlstrom KR, Nyberg L: Treatment of chronic stress in employees: subjective, cognitive and neural correlates. Scand J Psychol 2005, 46:395-402

38. Bergdahl J: [Psychosomatics. Affect based investigation and treatment of patients with psychosomatic problems][In Swedish). Svensk Rehabilitering 1999, 1:25-27.

39. Persson LAB-Å: [Affect integration. Manual to Affect School and Script Analysis][In Swedish]. 2003.

40. Arnstrom Johannessen C: [Affect School in primary care, a psychosomatic project][In Swedish]. 2002.

41. Williams JMGBT, Crane C, Hermans D, Raes F, Watkins E, Dalgeish T: Autobiographical memory specificity and emotional disorder. Psychological Bulletin 2007, 133:122-148.

42. Williams JTJK, Segal ZV, Soulsby J: Mindfulness-based cognitive therapy reduces over-general autobiographical memory in formerly depressed patients. Journal of Abnormal Psychology 2000, 109:150-155.

43. Greenberg L: Emotion-focused therapy. Clinical Psychology and Psychotherapy 2004, 11(3-16):

44. Angus LKKH: Margaret's story: An intensive case analysis of insight and narrative process change in client-centered psychotherapy. Insight in psychotherapy Washington, DC: Castonguay \& Hill; 2007

45. Bagby RM, Parker JD, Taylor GJ: The twenty-item Toronto Alexithymia Scale--I. Item selection and cross-validation of the factor structure. J Psychosom Res 1994, 38:23-32.

46. Bagby RM, Taylor GJ, Parker JD: The Twenty-item Toronto Alexithymia Scale--Il. Convergent, discriminant, and concurrent validity. $J$ Psychosom Res 1994, 38:33-40.

47. Bagby RM, Taylor GJ, Quilty LC, Parker JD: Reexamining the factor structure of the 20-item Toronto alexithymia scale: commentary on Gignac, Palmer, and Stough. J Pers Assess 2007, 89:258-64.

48. Taylor GJ, Bagby RM, Parker JD: The 20-Item Toronto Alexithymia Scale. IV. Reliability and factorial validity in different languages and cultures. J Psychosom Res 2003, 55:277-83.

49. Moriguchi Y, Maeda M, Igarashi T, Ishikawa T, Shoji M, Kubo C, et al.: Age and gender effect on alexithymia in large, Japanese community and clinical samples: a cross-validation study of the Toronto Alexithymia Scale (TAS-20). Biopsychosoc Med 2007, 1:7.

50. Parker JD, Bar-On R, Parker J: The handbook of Emotional intelligence. 2000

51. Martinez-Sanchez F: The Spanish version of the Toronto Alexithymia Scale (TAS-20). Clinica y Salud 1996, 7:19-32

52. Simonsson-Sarnecki M, Lundh LG, Torestad B, Bagby RM, Taylor GJ, Parker JD: A Swedish translation of the 20-item Toronto Alexithymia Scale: cross-validation of the factor structure. Scand J Psychol 2000, 41:25-30.

53. Arimura T, Komaki G, Murakami S, Tamagawa K, Nishikata H, Kawai K, et al: Development of the Structured Interview by the modified edition of Beth Isral Hospital Psychosomatic Questionnaire (SIBIQ) in Japanese Edition to evaluate alexithymia. Jpn J Psychosom Med 2002, 42:259-269.

54. Luminet O, Bagby RM, Taylor GJ: A multimodal investigation of emotional responding in alexithymia. Cognition and emotion 2004, 18:741-766.

55. Henry JD, Phillips LH, Maylor EA, Hosie J, Milne AB, Meyer C: A new conceptualization of alexithymia in the general adult population: 
implications for research involving older adults. J Psychosom Res 2006, 60:535-43.

56. Nishimura H, Komaki G, Igarashi T, Moriguchi Y, Kajiwara S, Akasaka T: Validity issues in the assessment of alexithymia related to the developmental stages of emotional cognition and language. Biopsychosoc Med 2009, 3:12.

57. Zigmond AS, Snaith RP: The hospital anxiety and depression scale. Acta Psychiatr Scand 1983, 67:361-70.

58. Bjelland IDAA, Haug TT, Neckelman D: The validity of the Hospital Anxiety and Depression Scale. An updated literature review. J Psychosom Res 2002, 52:69-77.

59. Lisspers J, Nygren A, Soderman E: Hospital Anxiety and Depression Scale (HAD): some psychometric data for a Swedish sample. Acta Psychiatr Scand 1997, 96:281-6

60. Wewers ME, Lowe NK: A critical review of visual analogue scales in the measurement of clinical phenomena. Res Nurs Health 1990, 13:227-36.

61. Thulesius H, Alveblom AK, Hakansson A: Post-traumatic stress associated with low self-rated well-being in primary care attenders. Nord J Psychiatry 2004, 58:261-6.

62. Krafft BNCNO, Pedersen C: [Stress reaction and welfare data- a population study][In Swedish]. Socialmedicinsk tidskrift 2004, 3:222-229.

doi: 10.1186/1751-0759-4-5

Cite this article as: Melin et al., Affect School for chronic benign pain patients showed improved alexithymia assessments with TAS-20 BioPsychoSocial Medicine 2010, 4:5

Submit your next manuscript to BioMed Central and take full advantage of:

- Convenient online submission

- Thorough peer review

- No space constraints or color figure charges

- Immediate publication on acceptance

- Inclusion in PubMed, CAS, Scopus and Google Scholar

- Research which is freely available for redistribution

Submit your manuscript at www.biomedcentral.com/submit
C) Biomed Central 